\title{
Designing an Internet Search Interface for Children
}

\author{
Nicholas Vanderschantz \\ Department of Computer Science \\ The University of Waikato \\ New Zealand \\ vtwoz@waikato.ac.nz
}

\author{
Annika Hinze \\ Department of Computer Science \\ The University of Waikato \\ New Zealand \\ hinze@waikato.ac.nz
}

\begin{abstract}
Interaction design and interface design research for children is of critical importance to ensure an educated future generation ready for the technology-focussed world. Significantly, the empirical studies that guide designers, developers, and technologists to support the production of suitable tools for children are under-represented in the scholarly literature. Using evidence from the literature combined with our own recent investigations, we provide a list of requirements for interfaces for children's educational information seeking. An interaction model and interface design are developed and tested with an expert heuristic evaluation and cognitive walkthrough.
\end{abstract}

Internet search engines, Children, Information seeking, Information search, Interface design, Children's interfaces

\section{INTRODUCTION}

Children are known to have difficulties effectively using the information technologies that are available to them (see for example Bilal, 2000, 2001, 2002; Large, Beheshti, \& Moukdad, 1999; Large, Beheshti, \& Rahman, 2002). Globally research has investigated children's information technology use in the classroom and the home. Analysis of the literature shows that recent work investigating children's internet searching is limited and much of that work has been undertaken with children in the United States of America (e.g. Bilal, 2000), the United Kingdom (e.g. Madden, Ford, Miller, \& Levy, 2006), and Europe (e.g. Gossen, 2013). Very little research is available to confirm nor contradict that the technologies, pedagogical practices, and findings from investigations in those large, predominantly Northern Hemisphere continents is appropriate or replicated for smaller nations and children in the Southern Hemisphere. This paper provides insight into New Zealand educational settings and recommends solutions that meet local values, traditions, practices and mind-sets. Future research will establish how these findings might translate globally.

We propose in this paper six requirements for systems or interfaces for children's information seeking. An interaction model and conceptual interface design for information search that is tailored to children's inquiry-based learning practices common in New Zealand classrooms and schools is introduced. Finally, we describe the
KidsQuestions prototype interface that we have developed based on these requirements.

The paper is structured as follows: the limited related work pertinent to our study is reviewed; we discuss the research we have undertaken to investigate the issues and experiences of children in New Zealand; we then use these findings to define the requirements for systems or interfaces for children's information seeking. The following section describes the conceptual design of an interaction model for children's information seeking based on these requirements. We introduce a proof-of-concept interface prototype and discuss the design decisions undertaken while designing. We conclude the paper with a discussion of the implications and the future work required to further this research.

\section{RELATED WORK}

The New Zealand education system encourages inquiry-based learning strategies by children across the schooling years. Inquiry-based learning is a teaching strategy that implements constructivist and socio-cultural theories and has been a central tenet of education for a number of years with a focus being present in the revised NZ curriculum published in 2007. Hook and Mills (2012) describe inquiry-based learning as an exploratory process that involves the children investigating their own questions, making discoveries, and testing their hypotheses. Hook and Mills continue suggesting that many NZ schools have adopted inquiry-based 
learning approaches and methods using traditional as well as ICT resources. It is not revealed in the literature to what extent and how information seeking takes place in today's classroom or home. This strengthens our argument for a need for exploration of an interaction model for children's information seeking that aligns with children's educational information seeking practices. The remainder of the Related Work discusses research into Information Communication Technology (ICT) in NZ education as well as the global research relevant to children's information search.

\subsection{ICT in NZ Education}

Much of the research pertaining to ICT in schools in $\mathrm{NZ}$ and further afield is predominantly influenced by investigation of high-level concepts and hardware or software resources. Recent hardware technology research has typically explored the use of contemporary technology devices in the classroom including tablet and mobile devices (e.g. ChanLin, Chou, \& Hung, 2015; Falloon, 2015; Falloon \& Khoo, 2014), interactive whiteboards (e.g. Hennessy \& London, 2013; Mellingsaeter \& Bungum, 2015), and voting tools (e.g. Moratelli \& DeJarnette, 2014; Premuroso, Tong, \& Beed, 2011; Scott, 2014). We identify reports in the literature of novel teaching of mathematics and programming using tablets and child-targeted software technologies (e.g. Beschorner \& Hutchison, 2013; Hegedus, 2013). Similarly there has been a focus on the use of software and devices for the creation of digital media such as video, photography, and digital storytelling (Tsai, Shen, \& Lin, 2015). Our own research has previously observed that children in NZ classrooms use this full range of technologies during their education (Timpany \& Vanderschantz, 2011; Vanderschantz et al., 2014a).

These studies primarily examine methods for distributing or delivering content or educational outcomes rather than during information seeking. Globally children are undertaking information seeking in formal educational settings and in the home. In NZ information seeking is formalised in education through the common pedagogical practices of inquiry-based learning. The breadth of information seeking tools and ICTs found in NZ classroom's and schools includes a variety of digital books, eBooks, and Internet resources, OPAC systems, and specific educational software (Timpany \& Vanderschantz, 2011).

The research reported in this paper focuses on children's successful and unsuccessful use of ICT for information seeking and use. Previous work in this area in NZ, and indeed the Southern Hemisphere, is limited. The most notable work in NZ was conducted by Moore (1995) in physical library spaces over two decades ago. Work with younger children than our target age range has been conducted in Australia (see for example Spink et al., 2010; Shuhidan et al., 2011). These examples from Australia have found similar search issues to those that our own related work (i.e. Vanderschantz \& Hinze, 2017) has identified.

\subsection{Children's Information Search Globally}

Cool (2004) gives a thorough review of the early studies of children's digital information seeking. Goals of these early studies were to investigate children's ability to use available, often commercial tools. The significant issue surrounding this literature is that most of what is known is dated and focuses on children searching for printed artefacts, (e.g., Moore, 1995), or using web search engines that utilise less robust search algorithms, (e.g., Bilal, 2000) than available today. More recent investigations have included larger samples of anonymous log data from naturalistic inquiries of information search logs, (e.g., Duarte Torres, Hiemstra, \& Serdyukov, 2010) from which information needs and purposes are difficult to ascertain. Recent studies have predominantly investigated search in the home, for example (Druin, Foss, Hutchinson, Golub, \& Hatley, 2010) rather than in an educational setting.

Studies have investigated the issues children experience when creating query constructions. These studies have included investigations of the types of queries, miss-spellings, repetition, and text entry strategies that children use (Bilal, 2000, 2001, 2002; Large, Beheshti, \& Moukdad, 1999; Large, Beheshti, \& Rahman, 2002; Schacter, Chung, \& Dorr, 1998). Large (2004) noted that for children to effectively use text based internet search engines (ISE's), the child was most often required to use keywords. Bilal (2000) observed that keyword searches by children were either too narrow or too broad for their information need. Large further reported that in 2004 few search engines accepted natural language question or sentence queries. As ISEs have developed, natural language queries have become suitable for some search situations. A further confounding issue with text-based search interfaces is that children are rarely able to develop synonyms or alternate words when their initial attempt fails (Bilal \& Kirby, 2002; De Vries, van der Meij, \& Lazonder, 2008). Query reformulation is the term given to the necessity to recreate a query when the first query has not produced the results the user requires. Query reformulation is an area that has received noteworthy discussion in the literature (i.e. Bilal, 1998; Bilal \& Kirby, 2002) with many issues remaining unresolved and scarce work having been conducted in the Southern Hemisphere.

The research suggests that the adult-oriented systems children use in the home and classroom, do not suit their information seeking needs because 
they require complex knowledge about search and query construction and often provide results that do not answer children's information needs (van der Sluis \& Van Dijk, 2010). Children often do not have the appropriate mental model to use these systems successfully. Researchers have counted the number of pages visited and revisited (Bilal, 2000, 2001, 2002, Large et al., 1999, 2002; Schacter et al., 1998) to understand what features of information display effect information search and information use. Children interacting with information presented within search engines and the resulting web pages has also been reviewed (McCrory et al., 2000; Schacter et al., 1998).

The standard response to this issue is the development of specialised child-centred information retrieval systems (Druin et al., 2003; Gossen, Nitsche, \& Nürnberger, 2012; Lingnau et al., 2010). The systems are often research-based prototypes and date visually very quickly and, naturally, do not receive on-going support akin to that of a commercial search engine. For a number of reasons, these dedicated child-centred systems are not used in NZ classrooms, and many are no longer available online. We advocate for system enhancements that support child appropriate information seeking behaviour within commonly available and regularly utilized systems instead of dedicated child-targeted solutions.

\section{OUR PREVIOUS CONTRIBUTIONS}

Little of the research reported in the related work was conducted in the Southern Hemisphere and therefore it is difficult to confirm that the issues and experiences of children match that of the Northern Hemisphere. Our ongoing research has focused on the information seeking needs of NZ school children who are between the age of 9 and 13 years old. We have undertaken a range of quantitative and qualitative studies including surveys of teachers and parents, interviews with teachers and children, and user observation studies with children. Our aim was to identify the resources that these NZ children use during their education, the issues that children experience using these tools, and how designers and developers may assist with information search and information seeking through the development of digital technologies. To date we have not conducted direct comparative studies with children both in NZ and further afield.

Key findings of this research have been that children in NZ classrooms and homes have access to and regularly use a range of ICT technologies from a very young age (Timpany \& Vanderschantz, 2011; Vanderschantz et al., 2014a). Some schools and homes in rural and lower socio-economic areas are identified to have more limited access to technologies including Internet access. We identified that Google is the most commonly used technology for children's information seeking as reported by both NZ teachers and children (Vanderschantz et al., 2014b). In our studies we have had no participant report use of dedicated children's specific ISE's, digital libraries, or other children's information retrieval systems. Teachers and children report difficulties creating search queries when using ISE's and OPAC's and a commonly held belief that keywords are the most appropriate search query construction for search.

We observed children (Vanderschantz \& Hinze, 2017) searching the internet and identified issues with search query formulation and query reformulation. In Vanderschantz \& Hinze (2017) we showed that children reformulated their search queries less often when they used natural language queries. Question based natural language queries were the most successful and align to the inquirybased learning common in NZ classrooms. The Related Searches offered by the search engine were seldom used by children in those studies. We also observed children having difficulty identifying which page they would like to visit and which pages they had already visited when reviewing a search engine results page list (Vanderschantz \& Hinze, 2017). It was observed that children were unsure where factual information displayed in pull-boxes or sidebars on ISE's came from and this compounded the effect of mistakenly visiting a website several times.

In summary, our work has identified three major difficulties for children: 1) constructing search queries, 2) identifying relevant information in search results lists, and 3) finding the information contained in web pages. The approach that we present in this paper begins to address difficulties 1 and 2.

\section{REQUIREMENTS}

Using the findings described above, we now propose six requirements for systems or interfaces for children's information seeking (see Table 1).

Table 1. Requirements for children's information seeking interfaces

\section{R1. Assistance with Natural Language} Query Construction

R2. Identification of Related Search and Query Construction

R3. Assistance with Mistaken and Repetitious Visiting of Websites

R4. Identification of Provenance of Information

R5. Align with Information Seeking Practices

R6. Appropriate Technology Environment

Here we discuss these recommendations in detail. 


\section{R1: Assistance with Natural Language Query Construction}

Section 2 and Section 3 of this paper has shown that children have limited search query construction strategies and children and teachers believe that keyword queries will best assist children using modern internet search engines. Modern ISE's successfully accept natural language queries and studies have shown these to be successful for children. Additionally, question-based search queries align with the inquiry-based learning frameworks common in NZ education. Therefore, we recommend interfaces that assist with the creation of natural language queries.

\section{R2: Identification of Related Search and Query Reconstructions}

In Section 2 and Section 3 we reported that children have limited search query construction strategies and struggle to adjust search queries when a query fails. Additionally, children lack the broad vocabulary required to create alternative search queries when required. We recommend that interfaces should help with the reformulation of queries through the improved identification and positioning of interface features such as related searches and the related searches relevance should be clarified for users.

\section{R3: Assistance with Mistaken and Repetitious Visiting of Websites}

Section 2 and Section 3 has shown that children struggle to successfully manage the information triage process during search. Interfaces should therefore assist with the clear identification of websites that have already been visited. A tool within the search interface that assists with planning of websites to visit may also assist with reducing repetitious visits.

\section{R4: Identification of Provenance of Information}

Our work discussed in Section 3 reported that children have difficulty identifying advertising, promotional, and sponsored content. Internet search engine interfaces should assist with the clear identification of content that is advertising or indeed remove advertising from ISE results intended for children. Highlighting where content is scraped from when this information is used within pull-boxes and sidebars will assist children.

\section{R5: Align with Information Seeking Practices}

Interfaces for children should encompass features and functions that align to reported information seeking practices, particularly inquiry-based learning frameworks identified as common for NZ education. Features that allow the user to explore their problem, use questioning techniques, and search planning should be at the fore of the tool so that it aligns to inquiry-based learning frameworks.

\section{R6: Appropriate Technology Environment}

Interfaces for children should be Internet accessible systems for desktop computers that are widely available and commercially supported. Neither standalone systems such as digital libraries and offline databases, nor dedicated children's search engines were discussed by teachers or children in investigations into NZ children's technology use (Timpany \& Vanderschantz, 2011; Vanderschantz et al., 2014b). Computer access was shown to be more highly available to children at all year levels than mobile technologies in both the home and the classroom (Vanderschantz et al., 2014a) and therefore we target desktop computers as our first priority in this research.

\section{INTERACTION MODEL}

An interaction model can be described as a model that is used during the development of a system or application in order to create a consistent user interaction or user flow. An interaction model should support users during their use of the system and should align with the user's mental model of how the system should work. Our interaction model is based on findings from interviews with NZ teachers and children (Vanderschantz et al., 2014b) and has been confirmed with observations of NZ children using Google (Vanderschantz \& Hinze, 2017). Here we describe our interaction model by means of a conceptual wireframe design. Figure 1 shows the interaction concept for a search engine search page, while Figure 2 shows the interaction concept for a search engine search results page (SERP).

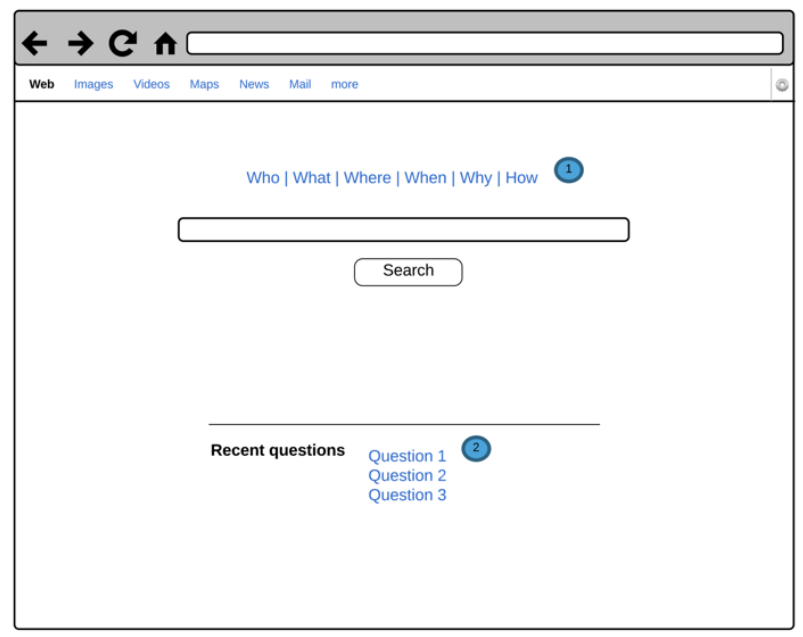

Figure 1 Wireframe visualising the interaction model for a Search Engine Search Page 


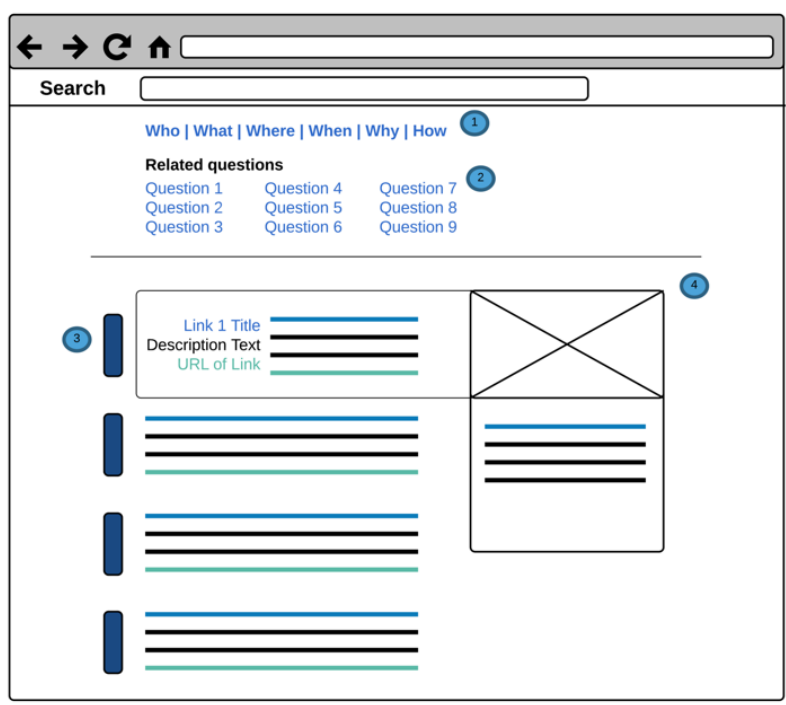

Figure 2 Wireframe visualising the interaction model for a Search Engine Results Page

We now discuss how these interaction designs address the requirements identified above.

To assist with query creation (R1), we propose three tools for the user that are designed to encourage the use of natural language queries. The three devices that we have implemented in our interaction model include Question Starter Ideas (see 1 in Figure 1 and 1 in Figure 2) of "what, where, when, why, how." These Question Starter Ideas are tools that are commonly introduced in early literacy education. Secondly, a set of Recently Asked Questions (see 2 in Figure 1) is supplied on the search page to inspire the user to consider constructing a query as a question. Additionally, the Related Searches commonly found in SERP's would be implemented as questions rather than keywords or short query strings (see 2 in Figure 2. Query expansion features common to search engines as a part of the search box would be retained.

To facilitate children's ability to locate the Related Searches (R2), we propose to give this feature high visual priority by placing these at the top of the SERP so that children are not required to scroll to locate the related searches (see 2 in Figure 2). These Related Searches should also be natural language queries and should promote use of question-based queries.

To improve the visual cues associated with representing visited websites (R3), we propose a simple Toggle Switch (see 3 in Figure 2) placed beside each result link. These switches have an unvisited and a visited state, which is a more graphic indication than the text colour change typically used by search engines for visited/unvisited indications.

We propose resolving the disconnection between the information in the sidebar and the link from which it is scraped from (R4) through visual design mechanisms which aim to align and highlight the content link (see 4 in Figure 2). We will not include any sponsored or advertising links in our prototype.

Identifying one's question is a fundamental part of many of the information seeking frameworks taught in NZ (R5). The inclusion of the Question Starter Ideas and Recently Asked Questions features may serve two purposes: 1) alleviating the difficulty for a child to move from a question they have developed (off computer) to a new way of searching for that question using keywords and topic words (on computer); and 2) ensuring children are developing questions and identifying their information need as opposed to searching without a clearly defined goal or question in mind. Throughout the interface, we propose repeating the use of the words "question" or "questions" to assist with reinforcing the use of question-based search queries. The starter text in the search box should explicitly direct the user to "Ask your question." Additionally, rephrasing text such as "Searches related to ..." to "You could ask a question about:" in order reinforce the use of natural language question.

Dedicated children's search engines were not discussed by teachers or children in our studies and therefore it may be more appropriate to implement enhancements to commonly available commercial systems rather than stand-alone online systems (R6). Investigating interface enhancements would ensure that these could be implemented by any commercial ISE manufacturer to assist children's information seeking.

\subsection{Scenario Walk Through}

We illustrate the interaction model here with a scenario walk through. A young user working on an Internet-connected device at school or at home considers their information need. They open the search page of the ISE in their browser of choice. The child would then enter a new search query based on their own evaluation of their need. Alternatively, the child might choose to use one of the Question Starter Ideas (see 1 in Figure 1) or Previously Asked Questions (see 2 in Figure 1) provided by the ISE to assist with their query formation. Once the user executes the query, the search results page is displayed to the user.

The user would then complete one of the following:

Option A: plan their search by triaging the result list using the Toggle Switches (see 3 in Figure 2), Option B: visit a link without first triaging the result list using the Toggle Switches, Option C: review content in a sidebar and follow the Sidebar Link (see 4 in Figure 2), Option D: reformulate query by hand or using the Related Questions feature (see 2 in Figure 2) or Question Starter Ideas device (see 1 in Figure 2). 


\section{PROOF-OF-CONCEPT INTERFACE PROTOTYPE}

In order to evaluate our interaction model for children's information seeking we developed the KidsQuestions prototype interface that implements our six requirements of our proposed interaction model. In line with R6, our intention was to test enhancements to an existing search engine (in this case Google) rather than propose a child-specific search engine.

Our intention is to test enhancements to already successful internet search engine interfaces and we expressly acknowledge no ownership of the design cues that have intentionally been developed to appear visually similar to either the Google aesthetic or that of similar ISE interfaces including Bing or Yahoo!. For this implementation of our recommendations and in order to test enhancements to an already successful internet search engine interface, many design cues were taken from the Google search engine. The decision to use features similar to Google for this first implementation is because this was the ISE most often reported by children and teachers in Vanderschantz et al., (2014b). Google's typographic choices such as typeface style, approximate type size, type colour as well as user interface features of buttons, button sizes, button colours and button placement were used to guide the design decisions made when developing our interface enhancements.

We are not affiliated with any search engine manufacturers and so we have altered the branding to ensure clear differentiation between our interface prototype and the Google search engine. To ensure we do not mislead test participants in future studies, we have used the logotype Questions in place of the Google logotype.

For reference, we show the search page and the results page in Google and KidsQuestions side-byside in Figure 2 and Figure 3 respectively.

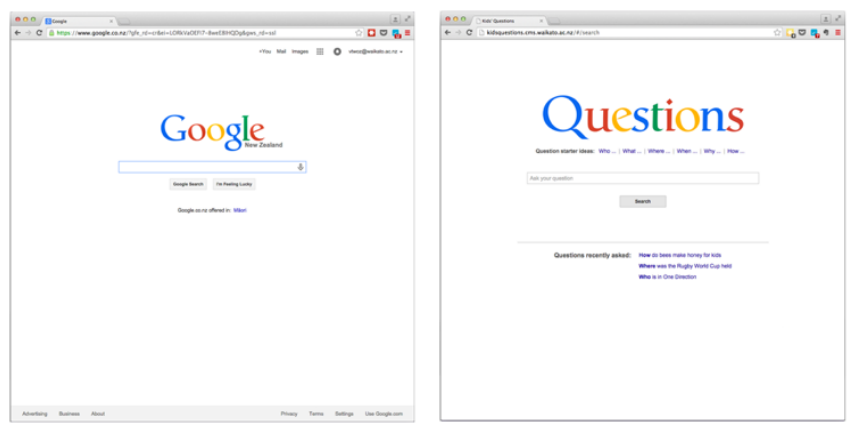

Figure 2 Comparison of the Google (left) and KidsQuestions Search Pages (right). Google and the Google logo are registered trademarks of Google Inc. used with permission.

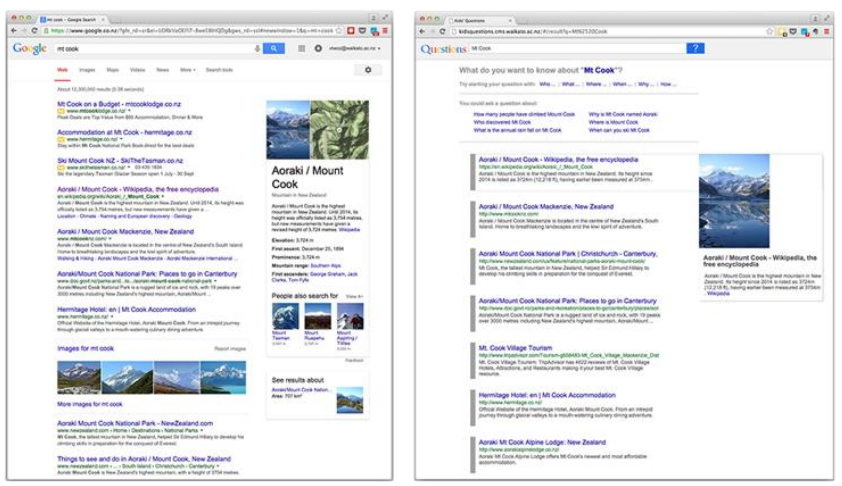

Figure 3 Comparison of the Google (left) and KidsQuestions search interface (right). Google and the Google logo are registered trademarks of Google Inc., used with permission.

This proof of concept implementation of our recommendations has been developed using design cues from Google. Because our recommendations are considered enhancements, these devices should be able to be implemented in any contemporary search engine.

\subsection{KidsQuestions Search Page}

Here we discuss the visual design decisions for the Search Page of the KidsQuestions interface (see Figure 4). This is the first page users will see when they interact with the search engine.

\subsubsection{Question Starter Ideas on Search Page}

The Question Starter Ideas (see A in Figure 4) are placed directly below the Questions logo. They are set in blue to indicate that they are interactive.

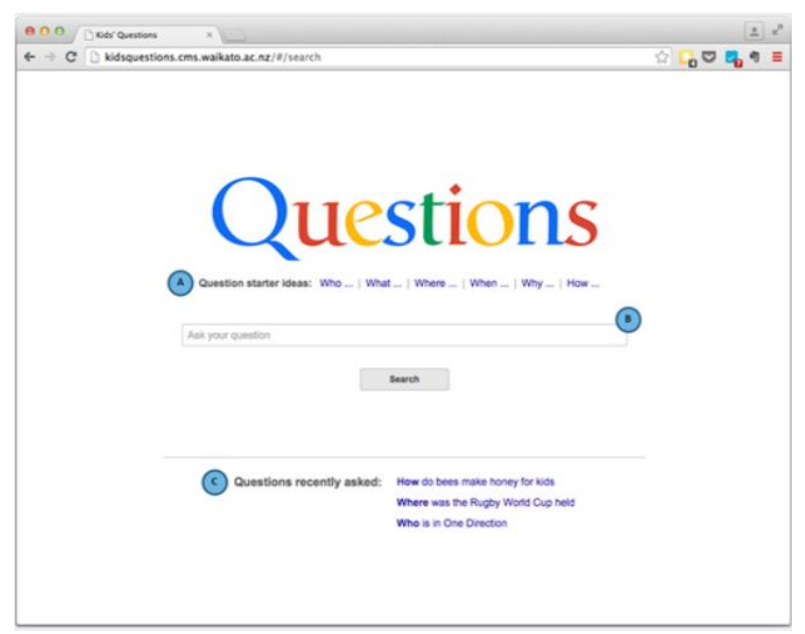

Figure 4 KidsQuestions Search page

All other clickable text links in the interface are also set in blue, which is a common internet design convention. The Question Starter Ideas are prefaced with the text "Question starter ideas:". When a user clicks one of these Question Starter Ideas the word clicked is placed as the first word in the search box. If there is already a question starter 
word as the first word in the search box, then this is replaced by the new question starter. If no question starter is present, but a text string is already in place, the new starter word is placed before the existing text string. Finally, if the search box is empty, then the question starter text is placed within the search box. Focus is given to the search box when a Question Starter Idea is clicked.

\subsubsection{Search Box on Search Page}

The search box (see B in Figure 4) is similar in location and size to the Google search box with the addition of the text "Ask your question." As with Google and other ISE's, we have implemented a drop-down query expansion service.

\subsubsection{Recently Asked Questions on Search Page}

The Recently Asked Questions (see C in Figure 4) are placed below the search box and search button. These are set in blue and the question word is bolded to reinforce the use of these words within queries typed by the user. When a user clicks one of these recently asked questions, the string is placed into the search box and awaits the user to edit the string or to fire the search. If the text is already present within the search box, the text string of the recently asked question that is clicked replaces this. Three questions are drawn from a database to present to the user each time this page is loaded.

\subsection{KidsQuestions Search Results Page}

Here we discuss the decisions made during the visual design of the SERP of the KidsQuestions search interface (see Figure 5). This is the page users are presented with after the user has fired a search.

As with text on the KidsQuestions Search Page, text and text links on the KidsQuestions SERP are also made larger than text used by other ISE's. Space between result list entries is larger for the KidsQuestions than other ISE's because of the increased visual space created by the implementation of the Triage Toggles.

The search bar is found at the top of the screen in a very similar nature to that implemented by many ISE's. We use a question mark as the icon for the search button on this screen as opposed to a magnifying glass to reinforce the idea of asking a question.

\subsubsection{Question Starter Ideas on SERP}

Below the search bar, we placed an instructional block designed to assist with reformulating search queries (see A in Figure 5). This block contains a reminder of the search constructed by the user with the question 'What do you want to know about “..."?'. The Question Starter Ideas device functions in exactly the same way as it does on the Search
Page but uses a slightly different text instruction "Try starting your question with:"

\subsubsection{Related Searches on SERP}

Below the instructional block we present the related searches block (see B in Figure 5). We instruct the user how to use these devices with the instruction: "You could ask a question about:". As per the Recently Asked Questions found on the Search Page, when one of these Related Questions is clicked the entire string is placed in the search box and the search box is given focus. This string replaces any text in the search box. We restrict the number of related questions to six simply to ensure that this content block does not push search results too far down the screen on small screen devices such as mobile tablets or small laptops.

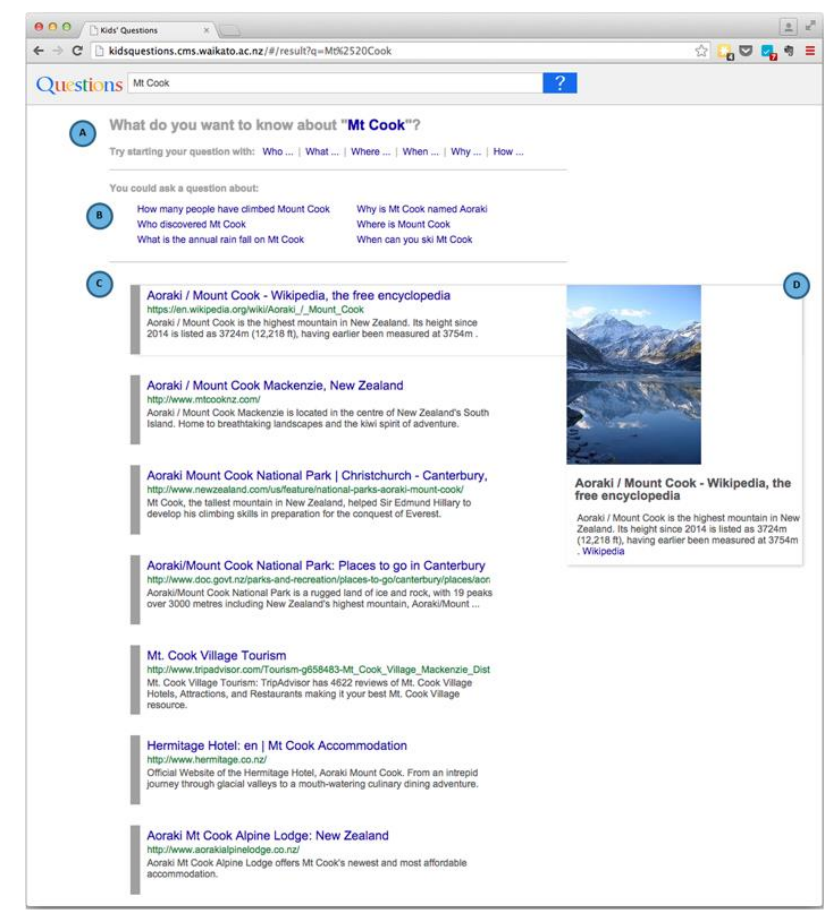

Figure 5 KidsQuestions search interface results page

\subsubsection{Triage Toggles on SERP}

The results list Triage Toggles (see $C$ in Figure 5) are implemented as small vertical bars placed to the left-hand side of each result list entry. Care was taken to develop a visual device that was large enough to be clickable, yet far enough away from the text to ensure no visual distraction when reading the link entry and no mistaken clicking of the toggle when a result link is the intended mouse target.

To encourage triage and pre-evaluation of websites to visit we have implemented a to-visit state (yellow) and to assist with remembering websites that contained information that was useful to the user we have also implemented a remember state (blue with the text "remember"). A null state is grey.

These four states can be seen in Figure 6 . 


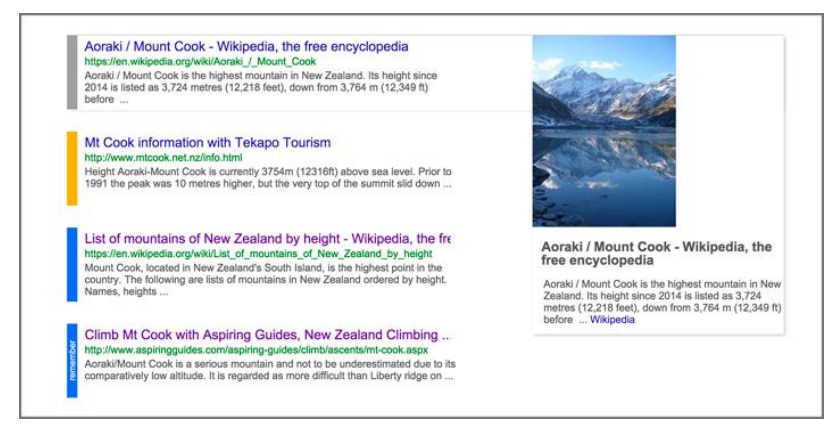

Figure 6 KidsQuestions triage Toggle Switches

\subsubsection{Sidebar on SERP}

While we have not implemented sidebar and pull bar content in our prototype in exactly the way that it is used by Google, we have implemented a sidebar which attempts to complete a small part of the service that Google's' sidebars and pull-boxes complete. An informational sidebar is created using content scraped from Wikipedia when the first entry in the SERP list is a Wikipedia entry (see D in Figure 5). The sidebar is populated with one or two images when extractable from the Wikipedia entry. The sidebar presents and formats the title, and the first paragraph of the Wikipedia entry. Creating a clear union of sidebar and entry in the SERP list for the site where information is scraped from was a central goal of the design of this device and thus the bordering of the SERP entry and the sidebar.

\subsubsection{Advertising on SERP}

We have removed all advertisements from our prototype to ensure unhindered results list review. Various search engines do an adequate job clarifying the difference between sponsored and non-sponsored advertisements, and there is a myriad of design considerations that may aid in clear differentiation for children. However, we have taken the stance that sponsored content is something that we would prefer not to include in an interface targeted at children, and thus it has been excluded from our prototype.

\section{EXPERT REVIEW}

Prior to conducting user studies with children, we conducted a usability, experience, and visual communication review of the KidsQuestions search interface with five experts. The goal of the expert review was to gain the first indication of any usability issues with the KidsQuestions search interface that required attention before deployment with a vulnerable youth audience.

\subsection{Method}

The expert review was conducted as a heuristic evaluation (Nielsen \& Molich, 1990) and brief cognitive walkthrough (Rieman et al. 1995) of typical usage scenarios for the KidsQuestions search interface. Five experts were invited to work with the system alongside the researcher in a talkaloud format and to individually review the interface. The experts were all familiar with the conceptual design and usability design of digital interfaces for varied target markets. Two of the experts who participated in this research are interface design researchers and practitioners, two of the experts are $\mathrm{HCl}$ and user experience designers and researchers, and the fifth participant is a children's designer and researcher.

During each review, the experts were first invited to use the system while observed by the researcher, next the researcher introduced the expert to any features they may not have interacted with, and finally, the researcher invited discussion of issues perceived by the experts. The experts tended to use a talk aloud protocol and answered the open questions of the researcher during the observations and after the utilization of the tool. As this was an informal invitation to offer insight, no recording of observations was made.

\subsection{Results}

The experts were generally positive about the visual design and the usability of the search engine and the enhancements proposed. No design flaws in the behaviour of the KidsQuestions search interface were identified by the experts. The expert review revealed a small number of visual and aesthetic improvement recommendations that we havesummarised in Table 2.

Table 2. Expert Recommendations

ER1. "I'm Feeling Lucky" button should be removed

ER2. Question Starter ideas should appear above the search box

ER3. Ellipses should be added to the Questions Starters

ER4. Related Searches should be natural language question queries not keyword queries

In response to these issues, we discuss here the following changes that were made to the KidsQuestions search interface.

\subsubsection{Expert Recommendation 1}

The "I'm Feeling Lucky" button was considered counter to the goals of this research as it did not encourage the use of Questions, did not facilitate the opportunity for query or topic expansion, nor did it encourage search results list triage and decision making. Additionally, in our study of Google described in (Vanderschantz \& Hinze, 2017) we did not see this button used by participants. Therefore, this button was removed from the interface and the search button made bigger and centred below the 
search box. See Figure 8 to review the design changes implemented based on this expert review.

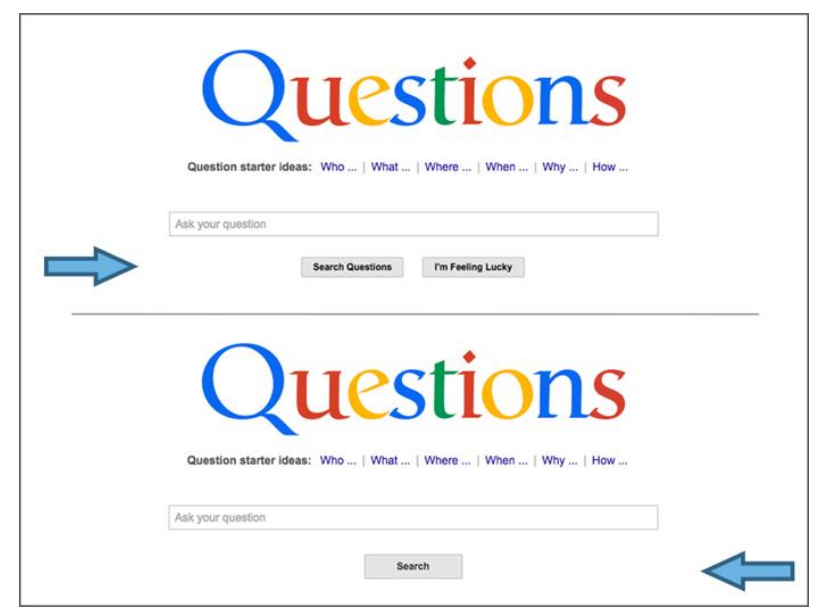

Figure 8. Removed "I'm Feeling Lucky" before (top) and after (bottom) expert review

\subsubsection{Expert Recommendation 2}

The placement of the Question Starter Ideas was questioned by a couple of the experts. It was considered that these could work either above or below the search box. We have considered both presentations and have moved the Question Starter ldeas above the search box improving the eye flow of the page. It is anticipated that by placing the Question Starter Ideas device above the search box these will be read and used before a query is entered by a user (see Figure 9).

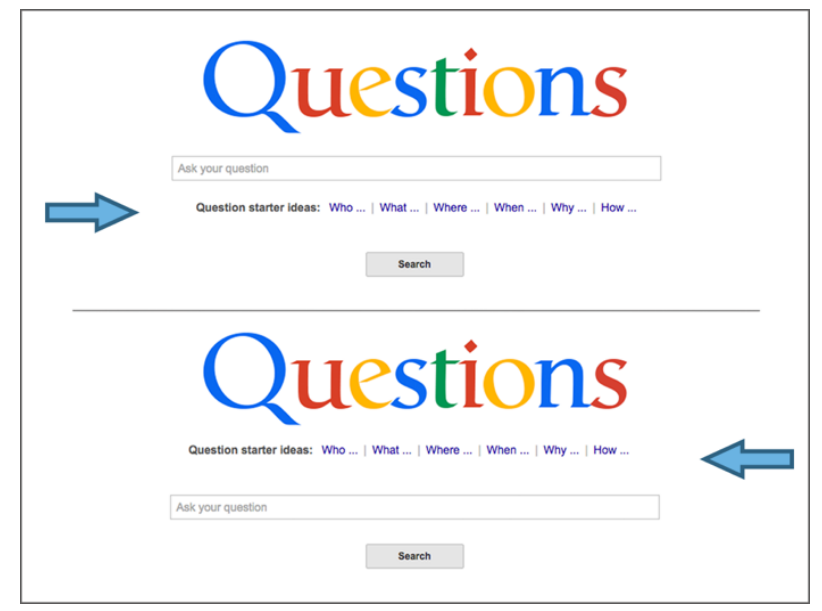

Figure 9. Question Starter Ideas before (top) and after (bottom) expert review

\subsubsection{Expert Recommendation 3}

One expert noted that the use of ellipses to indicate that the Question Starter Ideas are the start of a question might add further semantic meaning to this device. This small suggestion was considered a positive improvement to the interface and ellipses were added to the Question Starter Ideas to indicate the requirement for additional information provided by the user as well as to draw attention to the clickability of these devices (see Figure 10).

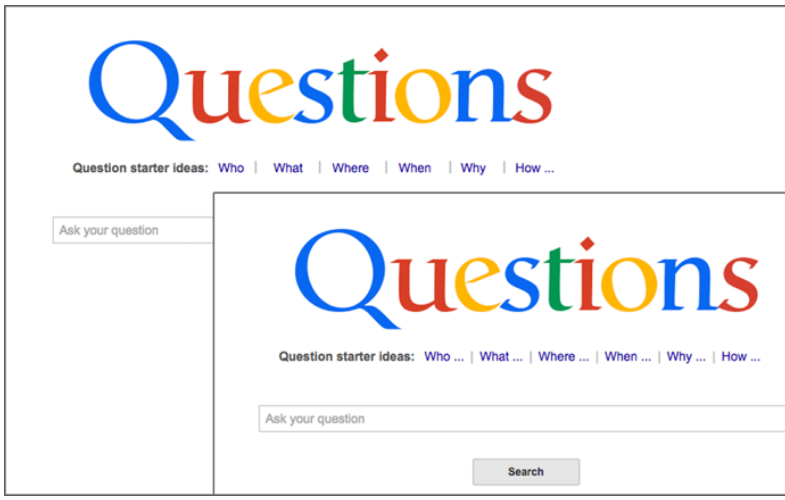

Figure 10. Question Starter Ideas ellipses before (back) and after (front) expert review

\subsubsection{Expert Recommendation 4}

Reviewers expressed that the inclusion of the Related Searches at the top of the results page was a positive design enhancement. However, the experts felt that the cognitive load required to rephrase a keyword query into a natural language query would be difficult. It was felt that the likelihood would be that a user would click the Related Search, which would then appear in the search box, and the query would be fired by the user without manual user adjustment of the query from a keyword to a question. Therefore, we abandoned our initial use of the Bing API from which we were receiving and including the relatedsearch entries and instead implemented the manual Question-based related-searches described in Section 6.2.

\section{DISCUSSION \& FUTURE WORK}

This interaction design is unique compared to the search engines discussed in the related work (see Section 2). Many researchers (e.g., Beheshti et al., 2010; Gossen, 2013; Reuter \& Druin, 2004) have developed child-specific search engines to address similar issues to those that we address in this study. Our own work (Vanderschantz et al., 2014b) found that students in New Zealand classrooms did not use child-specific search engines and thus, implementing enhancements within commercial ISE's may better serve NZ school children.

The goal of our research is not to develop a search engine that competes with other search engines, nor a child-specific search engine. We hypothesise that our interaction model and interface design enhancements could be implemented in any commercial search engine. For this investigation we chose Google to enhance because this was the search engine discussed by most children and teachers in our related study (Vanderschantz et al., 2014b). It would be equally possible to implement the features of our interaction model and our prototype search engine enhancements in Bing, Yahoo! or, any other internet search engine. 
Enhancing commonly used search engines will help to ensure that children become familiar with tools that they have access to and are expected to use at home and in later life. While the interaction model and interface design proposed in this paper is targeted at children in educational learning environments the non-intrusive nature of the enhancements may still be appropriate for daily use by adults. This differs from the typical systems described in the related work which target only children (e.g., Beheshti et al., 2010; Gossen, 2013; Reuter \& Druin, 2004) and would serve users for only a portion of their life. Future work will test the use of our interface prototype with adult users as well as children.

Our related investigations (see for example Vanderschantz \& Hinze, 2017) observed NZ children undertaking inquiry-based learning projects using commercial ISE's. These observations revealed the successes NZ children gained when using question-based natural language search queries. Assisting users, both adults and children, to understand the potential successes of natural language search query constructions may assist users in more successfully using ISE's. As an alternative tactic, we have taken the approach of enhancing ISE's to promote natural language query construction. Future work should also investigate the opportunities to support educators to understand the benefits for children in using natural language search queries.

Encouraging question-based natural language search queries aligns with the inquiry-based learning frameworks common in NZ (i.e. Kellow, 2006) and aligns with the common learning resources found on NZ classroom walls that are intended to encourage the use of thinking with questions. Our implementation of interface enhancements that encourage question-based natural language search is therefore situated within these common NZ frameworks. The information retrieval interfaces for children reported in the literature (e.g. Gossen et al., 2012; Lingnau et al., 2010) have not implemented design features that encourage natural language query constructions.

The pedagogies common to NZ classrooms are not restricted to NZ or the Southern Hemisphere and therefore this tool may prove useful globally. Kellow (2006) notes that many of the implementations of inquiry based learning frameworks in NZ are based on models developed outside of NZ including Action Learning (Gawith, 1988) and Big 6 (Eisenberg \& Berkowitz, 1990). Given this, our interaction model and resulting ISE enhancements that are based on the pedagogies common to NZ classrooms would likely still benefit children globally. Future work will test the use of this interface prototype outside of NZ.
We have focused our design on an interface prototype for a desktop computer. We expect that the features of our design would be equally suitable for small screen devices with some visual design adjustments needed at deployment on mobile device screens. Future work will assess small screen implementation and use of KidsQuestions.

\section{CONCLUSION}

This paper has provided a review of the need for unique design consideration for children in the New Zealand education system. We have shown that research pertaining to children's technology needs during their education is rapidly becoming dated and that investigations further afield than the US, UK, and Europe are missing from the literature. This paper outlines our initial design investigations for internet search interfaces developed in order to begin resolving this gap in the literature.

We propose six requirements for systems or interfaces for children's information seeking. An interaction model and conceptual interface design for information search based on these requirements is introduced. This design is tailored to children's inquiry-based learning practices common in New Zealand classrooms and schools. Further, we describe the KidsQuestions prototype interface that we have developed based on these requirements.

An expert heuristic review and cognitive walkthrough analysis of the interaction and interface design of our ISE suggests that no usability flaws were present in the prototype that we have implemented. The expert review revealed a small number of visual and aesthetic improvement recommendations that were implemented and discussed in the paper. This review reinforces our demonstration of the potential for the implementation of our six requirements for children's information seeking interfaces. Future work will provide empirical evidence of the success of this interface when used by children and adults.

We thus recommend implementation of our six requirements for children's information seeking interfaces by commercial ISE's and further testing by other scholars:

\section{R1: Assistance with Natural Language Query Construction}

R2: Identification of Related Search and Query Reconstructions

R3: Assistance with Mistaken Repetitious Visiting of Websites

R4: Identification of Provenance of Information

R5: Align with Information Seeking Practices

R6: Appropriate Technology Environment 


\section{REFERENCES}

Beheshti, J., Bilal, D., Druin, A., \& Large, A. (2010). Testing children's information retrieval systems: Challenges in a new era. In Proceedings of the American Society for Information Science and Technology (Vol. 47, pp. 1-4). USA: Wiley.

Beschorner, B., \& Hutchison, A. (2013). iPads as a Literacy Teaching Tool in Early Childhood. Online Submission, 1(1), 16-24.

Bilal, D. (1998). Children's search processes in using World Wide Web search engines: An exploratory study. In Proceedings of the Annual Meeting-American Society for Information Science (Vol. 35, pp. 45-53). London, England: Learned Information (Europe) Ltd.

Bilal, D. (2000). Children's use of the Yahooligans! Web search engine: I. Cognitive, physical, and affective behaviors on fact-based search tasks. Journal of the American Society for Information Science, 51(7), 646-665.

Bilal, D. (2001). Children's use of the Yahooligans! Web search engine: II. Cognitive and physical behaviors on research tasks. Journal of the American Society for Information Science and Technology, 52(2), 118-136.

Bilal, D. (2002). Children's use of the Yahooligans! Web search engine. III. Cognitive and physical behaviors on fully self-generated search tasks. Journal of the American Society for Information Science and Technology, 53(13), 1170-1183.

Bilal, D., \& Kirby, J. (2002). Differences and similarities in information seeking: children and adults as Web users. Information Processing \& Management, 38(5), 649-670.

ChanLin, L.-J., Chou, T.-L., \& Hung, W.-H. (2015). Bridging Volunteer Services and Mobile Teaching in the Tablet Reading Community. Libri, 65(4), 269-280.

Cool, C. (2004). Information-seeking behaviors of children using electronic information services during the early years: 1980-1990. In M. K. Chelton \& C. Cool (Eds.), Youth InformationSeeking Behavior: Context, Theories, and Models (pp. 1-35). Lanham, Maryland: Scarecrow Press.

De Vries, B., van der Meij, H., \& Lazonder, A. W. (2008). Supporting reflective web searching in elementary schools. Computers in Human Behavior, 24(3), 649-665.

Druin, A., Bederson, B., Weeks, A., Farber, A., Grosjean, J., Guha, M. ., ... Zhang, L. (2003). The International Children's Digital Library: Description and analysis of first use (Technical Report No. CS-TR-4433 UMIACS). University of Maryland.
Druin, A., Foss, E., Hutchinson, H., Golub, E., \& Hatley, L. (2010). Children's roles using keyword search interfaces at home. In Proceedings of the SIGCHI Conference on Human Factors in Computing Systems (pp. 413-422). New York, NY, USA: ACM.

Duarte Torres, S., Hiemstra, D., \& Serdyukov, P. (2010). An Analysis of Queries Intended to Search Information for Children. In Proceedings of the Third Symposium on Information Interaction in Context (pp. 235-244). New York, NY, USA: ACM.

Eisenberg, M. B., \& Berkowitz, R. E. (1990). Information problem-solving: the Big Six Skills approach to library \& information skills instruction. Norwood, New Jersey: Ablex Publishing Corporation.

Falloon, G. (2015). What's the difference? Learning collaboratively using iPads in conventional classrooms. Computers \& Education, 84, 62-77.

Falloon, G., \& Khoo, E. (2014). Exploring young students' talk in iPad-supported collaborative learning environments. Computers \& Education, 77, 13-28.

Gawith, G. (1988). Action learning: Student guide to research and information skills. Auckland, New Zealand: Longman Paul Limited.

Gossen, T. (2013). Towards Appropriate Search User Interfaces for Children. MagdeburgerInformatik-Tage 2. Doktorandentagung 2013 (MIT 2013), 7.

Gossen, T., Nitsche, M., \& Nürnberger, A. (2012). Knowledge journey: a web search interface for young users. In 6th Symposium on HumanComputer Interaction and Information Retrieval (pp. 1-10). New York, NY, USA: ACM.

Hegedus, S. (2013). Young Children Investigating Advanced Mathematical Concepts With Haptic Technologies: Future Design Perspectives. The Mathematics Enthusiast, 10(1/2), 87-107.

Hennessy, S., \& London, L. (2013). Learning from International Experiences with Interactive Whiteboards. OECD Publishing, Paris, 89.

Hook, P., \& Mills, J. (2012). Inquire2Learn Article HookED Wiki. Retrieved 12 April 2016, from http://pamhook.com/wiki/Inquire2Learn_Article

Kellow, J. M. (2006). Inquiry Learning in an ICT-rich Environment (Technical Report). Retrieved from http://inquiringmind.co.nz/FinalResearchReportJ MK.pdf

Large, A. (2004). Information seeking on the Web by elementary school students. In M. K. Chelton \& C. Cool (Eds.), Youth information-seeking behavior: Theories, models, and issues (pp. 293-319). Lanham, Maryland: Scarecrow Press. 
Large, A., Beheshti, J., \& Moukdad, H. (1999). Information Seeking on the Web: Navigational Skills of Grade-Six Primary School Students. In Proceedings of the American Society for Information Science and Technology (Vol. 36, pp. 84-97). USA: ASIS\&T.

Large, A., Beheshti, J., \& Rahman, T. (2002). Gender differences in collaborative web searching behavior: an elementary school study. Information Processing \& Management, 38(3), 427-443.

Lingnau, A., Ruthven, I., Landoni, M., \& Van der Sluis, F. (2010). Interactive search interfaces for young children - the PuppyIR approach. In Proceedings of Advanced Learning Technologies (ICALT), 2010 IEEE 10th International Conference on (pp. 389-390). Sousse: IEEE.

Madden, A. D., Ford, N. J., Miller, D., \& Levy, P. (2006). Children's use of the internet for information-seeking: What strategies do they use, and what factors affect their performance? Journal of Documentation, 62(6), 744-761.

McCrory Wallace, R., Kupperman, J., Krajcik, J., \& Soloway, E. (2000). Science on the Web: Students online in a sixth-grade classroom. The Journal of the Learning Sciences, 9(1), 75-104.

Mellingsaeter, M. S., \& Bungum, B. (2015). Students' use of the interactive whiteboard during physics group work. European Journal of Engineering Education, 40(2), 115-127.

Moore, P. (1995). Information problem solving: A wider view of library skills. Contemporary Educational Psychology, 20(1), 1-31.

Moratelli, K., \& DeJarnette, N. K. (2014). Clickers to the Rescue. The Reading Teacher, 67(8), 586593.

Nielsen, J., \& Molich, R. (1990, March). Heuristic evaluation of user interfaces. In Proceedings of the SIGCHI conference on Human factors in computing systems (pp. 249-256). ACM.

Premuroso, R. F., Tong, L., \& Beed, T. K. (2011). Does using clickers in the classroom matter to student performance and satisfaction when taking the introductory financial accounting course? Issues in Accounting Education, 26(4), 701-723.

Reuter, K., \& Druin, A. (2004). Bringing together children and books: An initial descriptive study of children's book searching and selection behavior in a digital library. In Proceedings of the American Society for Information Science and Technology (Vol. 41, pp. 339-348). USA: Wiley.

Rieman, J., Franzke, M., \& Redmiles, D. (1995, May). Usability evaluation with the cognitive walkthrough. In Conference companion on Human factors in computing systems (pp. 387388). ACM.

Schacter, J., Chung, G. K. W. K., \& Dorr, A. (1998). Children's Internet searching on complex problems: performance and process analyses. Journal of the American Society for Information Science, 49(9), 840-849.

Scott, V. (2014). Clicking in the Classroom: Using a Student Response System in an Elementary Classroom. New Horizons for Learning, 11(1).

Mohamed Shuhidan, S., Macauley, P., \& Reynolds, S. (2011). Internet seeking by Australian school children: teachers' perspectives. In Internet Technologies \& Society (pp. 159-165). IADIS Press.

Spink, A., Danby, S., Mallan, K., \& Butler, C. (2010). Exploring young children's web searching and technoliteracy. Journal of documentation, 66(2), 191-206.

Timpany, C., \& Vanderschantz, N. (2011). Learning outcome dependency on contemporary ICT in the New Zealand middle school classroom. In Proceedings of the 12th Annual Conference of the New Zealand Chapter of the ACM Special Interest Group on Computer-Human Interaction (pp. 65-72). ACM.

Tsai, C.-W., Shen, P.-D., \& Lin, R.-A. (2015). Exploring the effects of student-centered projectbased learning with initiation on students' computing skills: A quasi-experimental study of digital storytelling. International Journal of Information and Communication Technology Education (IJICTE), 11(1), 27-43.

van der Sluis, F., \& Van Dijk, E. M. A. G. (2010, July 23). A closer look at children's information retrieval usage: Towards child-centered relevance [Conference or Workshop Paper].

Vanderschantz, N., \& Hinze, A. (2017). A study of children's search query formulation habits. In Proceedings of the 31st British Computer Society Human Computer Interaction Conference, UK: ACM, New York, NY.

Vanderschantz, N., Hinze, A., \& Cunningham, SJ. (2014a). Current educational technology use for digital information acquisition by young New Zealand children. In Proceedings of the 37th Australasian Computer Science Conference Volume 147. (pp. 125-134). Australian Computer Society, Inc.

Vanderschantz, N., Hinze, A., \& Cunningham, SJ. (2014b). "Sometimes the internet reads the question wrong": Children's search strategies \& difficulties. In Proceedings of the SeventySeventh ASIST Annual Meeting (Vol. 51). Seattle, WA: Wiley. 